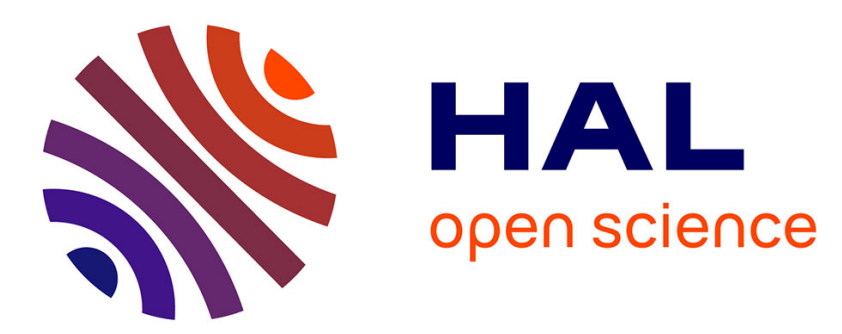

\title{
Dimensions réelles et dimensions imaginaires des dieux d'Egypte: les statues secrètes du temple d'Opet à Karnak
}

\author{
Claude Traunecker
}

\section{- To cite this version:}

Claude Traunecker. Dimensions réelles et dimensions imaginaires des dieux d'Egypte: les statues secrètes du temple d'Opet à Karnak. KTĖMA Civilisations de l'Orient, de la Grèce et de Rome antiques, 2004, 29, pp.51-65. halshs-00003816

\section{HAL Id: halshs-00003816 \\ https://shs.hal.science/halshs-00003816}

Submitted on 4 Feb 2005

HAL is a multi-disciplinary open access archive for the deposit and dissemination of scientific research documents, whether they are published or not. The documents may come from teaching and research institutions in France or abroad, or from public or private research centers.
L'archive ouverte pluridisciplinaire HAL, est destinée au dépôt et à la diffusion de documents scientifiques de niveau recherche, publiés ou non, émanant des établissements d'enseignement et de recherche français ou étrangers, des laboratoires publics ou privés. 


\title{
Extrait de KTEMA 29, 2004, p. 51-65.
}

\section{Dimensions réelles et dimensions imaginaires des dieux d'Egypte: Les statues secrètes du temple d'Opet à Karnak}

\begin{abstract}
RÉSUMÉ - L'iconographie égyptienne autorise la juxtaposition d'images d'échelles différentes dans la même composition. L'échelle peut être un indicateur de la hiérarchie des personnages et des fonctions. Cependant hommes et dieux sont représentés à la même échelle. Les parois des cryptes des temples tardifs portent des représentations des statues de culte avec des indications de leurs dimensions et du matériaux dont elles sont faites. Dans les cryptes, encore inédites, du temple d'Opet à Karnak certaines de ces images sont représentées à l'échelle $1 / 1$ de sorte qu'il est possible d'identifier les points de mesure pris en considération. Dans le décor de la salle de culte attenante à la crypte, une image d'Osiris, dépourvue d'indication de matière et de dimension, se distingue par sa taille de l'ensemble des représentations divines de cette salle. Elle se réfère ainsi, discrètement et uniquement pour les prêtres de haut rang, à la statue sacro-sainte cachée dans la crypte.
\end{abstract}

Zusammenfassung - . In der ägyptischen Bildkunst können verschiedene Maßstäbe nebeneinander verwendet werden. Der Maßstab ermittelt dem Betrachter Auskunft über Rang und Funktion der Dargestellten. Dennoch kommen Götter und Menschen im selben Malßstab vor. An Kryptenwänden der späteren Tempel sieht man Kultbilder mit Angebung ihrer Dimensionen und Materialien. In den noch unveröffentlichen Krypten des Opettempels in Karnak erscheinen die Götterbilder im Maßstab 1:1, woraus Anhaltpunkte für die Bildgestaltung gewonnen werden. In einem Nachbarsaal wird ein Osirisbild grösser als die anderen Figuren dargestellt. Es bezieht sich auf die geheime Kultstatue der Gottheit in einer Tempelkrypte.

\section{INTRODUCTION}

"Cher collègue, nous venons de trouver un sphinx monumental superbe. Connaissant votre intérêt pour ce type d'objet, nous serions heureux de le soumettre à votre sagacité lors d'un de vos prochains passages à Tanis». Le mot était accompagné d'une photo d'un sphinx à peine dégagé de sa gangue de terre. Cette lettre était adressée à un égyptologue résidant au Caire par Pierre Montet, le fouilleur de Tanis et Directeur de l'Institut d'égyptologie de Strasbourg. Elle produisit son effet et quelques jours plus tard, le collègue arriva à Tanis. Pierre Montet lui fit visiter les dernières fouilles, commenta les découvertes récentes, présenta ses projets, mais point de sphinx monumental! Finalement, le visiteur s'étonnant auprès du fouilleur strasbourgeois de ne pas voir le fameux sphinx, Pierre Montet s'exclama: "Ah, excusez-moi j'allais l'oublier! je l'ai sur moi, le voici» et, devant son collègue médusé, il sortit de sa poche une boîte d'allumettes, l'ouvrit et en sortit une boule de coton qui contenait un minuscule sphinx en faïence d'une facture parfaite. Cette anecdote qui courait dans l'égyptologie des années soixante montre combien l'art égyptien est hors échelle. La stylisation et la rationalisation des formes sont telles que toutes les échelles absolues 
sont possibles, de quelques millimètres à plusieurs mètres. Les statues colossales, grandeur nature ou simplement les minuscules statuettes portées en amulettes sont construites sur le même système de proportions. La sobriété des postures, voire des costumes permet cette multiplication d'échelles propre à l'art égyptien ${ }^{1}$.

Le problème de la taille des dieux peut s'aborder de trois manières différentes selon les sources: les dimensions relatives des dieux et des hommes dans l'iconographie, les tailles de dieux selon les textes et enfin, la taille des dieux dans leurs apparences terrestres que sont les statues de culte. Après avoir esquissé très brièvement les deux premières approches, je présenterai un dossier nouveau traitant les dimensions des statues de culte du temple d'Opet à Karnak selon des inscriptions inédites.

TAILlE DES DIEUX, TAILLE DES HOMMES, TAILLE DES IMAGES.

Dans la représentation en deux dimensions, comme d'ailleurs dans la statuaire, il est possible de juxtaposer ces échelles différentes. Il est généralement affirmé que le personnage le plus grand de taille est le plus important. Ainsi, dans les scènes de la fête-sed de Niouserrê $\hat{e}^{2}$, la taille du roi est le double de celle des officiants, serviteurs et assistants, qui s'affairent autour de lui. Dans les scènes de batailles, le roi sur son char ainsi que son attelage sont de taille héroïque par rapport à la masse des malheureux ennemis bousculés et piétinés par Pharaon ${ }^{3}$. Dans la statuaire, les groupes présentent parfois des hiérarchisations de tailles, l'exemple le plus célèbre étant les colosses de Memnon, où les princesses et reines d'Amenhotep III sont présentes, adossées au trône et guère plus grandes que le mollet royal ${ }^{4}$.

Ce phénomène est bien connu et a été largement décrit et étudié 5 . Dans les peintures et les basreliefs, il est des cas de représentations complexes (par exemple scènes de pressage par torsion) où les protagonistes sont représentés à des échelles différentes pour des raisons de composition ${ }^{6}$.

Dans l'ensemble, la règle de l'isocéphalie est respectée. Il faut mettre à part les conséquences de l'isocéphalie lorsque cohabitent dans une même image des personnages debout et assis. Dans ce cas, la taille des personnages assis est nettement plus grande. Une peinture thébaine, par exemple, conservée au Musée de Bâle, montre le couple de défunts assis, l'épouse légèrement plus petite que son mari, servi par une dame de la famille debout. L'officiant est isocéphale à l'épouse ${ }^{7}$.

(1) Voir Emma Brunner-Traut "Aspektive» dans Lexikon der Ägyptologie I, 1973, col. 474-488, eadem "Aspective" dans Heinrich SCHAfer Principles of Egyptian Art édition anglaise par John BAINES, Oxford 1974, p. 420-446;

(2) H. Schafer, o.c., p. 232, fig. 248: F. W. von Bissing, H. Kees, Das Re-Heiligtum des Konigs Ne-Woser-Re (Rathures) 2. Leipzig 1923, pl. 16.

(3) Scène de bataille du coffret de Toutankhamon: H. W. MüLLER, dans Les Pharaons III, L'empire des conquérants, l'Univers des Formes, 1979, p.177, fig.107; voir aussi p. 116 et 72. R. Schulz und M. Seidel édit. Die Welt der Pharaonen Cologne 1997, p. 163, fig. 27

(4) P. Barguet dans Les Pharaons II, L'empire des conquérants, l'Univers des Formes, 1979, p. 40. R. Schulz und M. SEIDEL O.C., p. 188, fig. 77.

(5) C. VANDERSLEYEN, «Les proportions relatives des personnages dans les statues-groupes», CdE XLVIII, No. 95, 1973, p. 13-25.

(6) H. Schafer, o.c., p. 231, fig. 247: 47); N. de G. Davies, The tomb of Puyemrê at Thebes, New York 1922. pl. 12. Voir aussi pour une version analogue à l'Ancien Empire R. DAvid, Les trésors de l'Égypte, Princesse Paris, 1980, pl. 18 (mastaba de Nefer).

(7) A. Wiese, Antikenmuseum Basel, Die ägyptische Abteilung, Mainz 2001, p.108, fig. 33 ( ${ }^{\circ} 67$ ). 
L'analyse de la taille des images ne doit pas dispenser de la lecture des textes. L'importance du bénéficiaire de l'objet (stèle décor) est indéniable, mais il arrive que le texte signale, voire représente, l'auteur de la stèle ou du relief ${ }^{8}$.

En ce qui concerne les dieux, nous constatons peu de discrimination par la taille. La grande majorité des représentations divines font partie de scènes d'offrandes montrant un roi officiant devant les dieux (pl. Ia.) Là, la règle d'isocéphalie est très largement respectée ${ }^{9}$. Il en va de même pour les documents mettant face à face un officiant non royal et les dieux ${ }^{10}$. La différence de statut du particulier et du roi vis-à-vis des dieux ne s'exprime pas dans les rapports de dimensions des images.

Les différences d'échelle d'images divines ont toujours des origines fonctionnelles. Ainsi la célèbre représentation de la Vache Céleste (pl. Ib.) ${ }^{11}$ juxtapose diverses approches métaphoriques du ciel: ventre d'une vache, fleuve céleste, ou encore toit soutenu par quatre étais. Sous une grande vache, glissent de petites barques solaires, Chou, le dieu de l'espace, soutient le ventre de l'animal, dont les quatre pattes, faisant fonction d'étais célestes, sont soutenus par les huit dieux traditionnels affectés à cette tâche. Les différences d'échelle de cette représentation résultent de la rationalité fonctionnelle de la composition, sorte d'icône informative de diverses traditions. Signalons pour terminer les cas où des images divines ont été rajoutées à d'anciennes scènes et sont donc, pour des raisons simplement matérielles, plus petites que les anciennes ${ }^{12}$.

Nous sommes là dans une problématique simple de composition et répartition iconographiques. Le but poursuivi est informatif et est hors de la nature des dieux. Leurs icônes sont à l'image et à l'échelle humaines.

Il en va autrement dans les textes: il n'est pas rare de trouver des mentions chiffrées de la taille des dieux ${ }^{13}$ : un hymne du temple d'Opet à Karnak accrédite Amon d'une taille fabuleuse de 2080 coudées $^{14}$.

\section{LE TEMPLE D'OPET À KARNAK}

Le temple d'Opet est situé dans l'angle sud-ouest de l'enceinte d'Amon. L'édifice actuel a été construit sous le règne de Ptolémée VIII Evergète $\mathrm{II}^{15}$. Il remplace un monument plus ancien remontant à l'époque éthiopienne ${ }^{16}$. Seul le décor des trois salles de culte et de la salle des offrandes

(8) Stèle de Montououser du Metropolitan Museum de New-York: P. Dorman, L'Egypte et le Proche Orient, Gründ, Paris 1987, p. 33. Moutououser est représenté assis. Devant lui, l'auteur et initiateur du document, son fils Intef est représenté mais en petite taille. Cette situation rappelle celle des orants dédicants des tableaux religieux occidentaux du XVe siécle.

(9) Par exemple R. David, o.c., pl. 35, 36, 38 (scènes du temple d'Abydos), pl. 131 (pl. 131, temple de Kom Ombo); Les Pharaons II, L'empire des conquérants, l'Univers des Formes, 1979, p. 71 (Chapelle peinte de Hathor)

(10) Par exemple R. David, o.c., pl. 107 (tombe de Pachedou); A. WIESE, o.c., p. 147 stèles 104; Les Pharaons III, L'Egypte du crépuscule, l'Univers des Formes, 1980, p. 117, pl. 103, voir aussi p. 115, 116, 118 avec des variantes.

(11) A. Piankoff, The Shrines of Tut-Ankh-Amon, p. 142, fig. 46; Erik HoRnung Das Grab Sethos' l. photographiert von Harry Burton, Artémis Zurich, 1991, p.220-221.

(12) C. TRAUNECKer, "Manifestations de piété personnelle à Karnak» dans Bulletin de la Société Française d'Egyptologie 85, 1979, p. 22-31; Rajout d'une image divine: HELCK, Die Ritualszenen auf die Umfassungsmauer Ramses II in Karnak, Abbildungen, p. 87; rajout d'un officiant: P. BARGUET, Le temple d'Amon-Rê, pl. xvii [B], p. 113-114 note 6.

(13) E. Chassinat, Le mystère d'Osiris au mois de Khoiak, Le Caire 1966, p. 279, note 7,

(14) C. de Wit, Opet I, 113, col. 12. Grande taille d'Osiris enfanté à Thèbes: Chassinat, Le mystère d'Osiris au mois de Khoiak I, p.279, note 7; Dendara II, p. 101, lg. 3: «Osiris de Thèbes de 4 coudées, 6 palmes et 3 doigts» soit 4,69 m.

(15) an 164, puis 145-116 avant J.-C. G. HöLBL, Geschichte des Ptolemaerreiches, 1994, p. 137-145, 177-182; M. Minas, Die Dekorationstätigkeit von Ptolemaios VI. Philometor und Ptolemaios VIII. Euergetes II. an ägyptischen Tempeln (Teil 1), OLP 27, 1996, p. 51-78.

(16) Taharqa (690-664 avant J.-C), La XXVe dynastie dite éthiopienne a régné sur l'Egypte de 716 à 656 avant J.-C. 
a été achevé. Il porte les noms de Ptolémée VIII Evergète II et de ses épouses Cléopâtre II et III. Les salles annexes (la salle hypostyle, l'escalier, les salles et couloirs nord et sud) sont restées anépigraphes sauf le couloir nord (salle des étoffes). Le décor des faces extérieures du temple est inachevé: seuls ont été sculptés l'encadrement de la porte principale, sous Ptolémée XII Néos Dionysos, et, sous Auguste, le soubassement et une partie de la façade sud. Hormis le décor des cryptes, l'ensemble des textes du temple a été publié par Constant de Wit ${ }^{17}$. Un relevé architectural a été effectué par mes soins, mais est resté en grande partie inédit ${ }^{18}$. L'édifice est exceptionnel, tant par sa nature que par sa forme ${ }^{19}$.

De par sa nature, il appartient à la catégorie rare des temples mythologiques. Il est consacré, non pas à une divinité ordinaire résidant dans son temple, mais à un moment d'un mythe, pris dans un ensemble d'ana ${ }^{20}$. A cette catégorie appartiennent, entre autres, le temple d'Isis à Dendara (naissance d'Isis) et les mammisis (naissance de l'enfant divin local). Le temple d'Opet commémore plusieurs moments des Osiriana: la naissance d'Osiris, sa résurrection, la conception et la naissance d'Horus, et finalement sa reconnaissance comme l'héritier d'Amon, dieu de la royauté. Opet, une figure d'hippopotame-mère semblable à Touéris, nest pas proprement parler une déesse mais la simple mise en image du concept de naissance. Ici cette naissance est double car elle concerne à la fois Osiris, né à Thèbes selon les Osiriana locaux, et Horus, l'enfant divin.

De par sa forme, le temple d'Opet est unique (pl. IIa). Trois sanctuaires sont disposés en croix autour d'une salle des offrandes centrale. Dans le petit sanctuaire axial (salle X) était vénérée l'image de culte d'Opet; au nord, une salle de culte était consacrée à Osiris et à sa résurrection (salle VIII). Enfin, en face, au sud, une seconde salle de culte était dédiée à Isis-mère divine et à son enfant Horus (salle IX).

À lavant de ce groupe cultuel, une salle hypostyle (deux colonnes hathoriques) commande quatre espaces de service: au nord-ouest, l'escalier, au nord-est, la salle dite des étoffes (salle V) ${ }^{21}$, au sud-ouest, la salle II (réserve des vases sacrés selon Rochemonteix) et au sud-est, les salles III et IV.

L'idée forte s'exprimant dans l'architecture du temple est la dualité de sa mythologie: au nord, Osiris renait (salle et cryptes nord); au sud Isis, mère divine, prend soin d'Horus l'enfant, considéré comment l'héritier de la royauté amonienne.

(17) C. de WIT, Les inscriptions du temple d'Opet à Karnak, Bibliotheca Aegyptiaca XI et XII, Bruxelles, 1958 et 1962. Bibliographie dans PM II 2, 1971, p. 244-252.

(18) C. Traunecker, Les cryptes du temple d'Opet à Karnak. Mémoire de l'Ecole Pratique des Hautes Etudes (direction J. Yoyotte) 1975, 331 pages, 139 figures et 7 planches, inédit. Le plan a été réalisé avec l'aide de Pierre Anus. Voir aussi M. AzIM «A propos du pylône du temple d'Opet à Karnak», Karnak VIII, p. 51-80. Emmanuel Laroze, architecte au Centre Franco-Egyptien de Karnak prévoit une étude architecturale du temple. Je le remercie vivement d'avoir bien voulu vérifier sur place les dimensions des images de la salle de culte nord.

(19) C. Traunecker, "Cryptes connues et inconnues des temples tardifs" dans Bulletin de la Société Française d'Egyptologie, 129, 1994, p. 21-45.

(20) Sur cette terminologie, voir C. Traunecker, Les dieux de l'Egypte, Que Sais-je, 1996, p. 96. Le terme de Mythe, souvent employé au singulier avec une majuscule rend très mal compte de la réalité égyptienne. A part quelques rares exceptions, nous sommes en présence de textes allusifs, courts, variés et souvent paradoxaux. Le "Mythe" d'Osiris tel qu'il est souvent "raconté» dans les manuel est une mise en forme occidentale due à Plutarque. Les sources égyptiennes sont beaucoup plus complexes et diverses. Je propose d'utiliser la terminaison -ana, employée par les historiens de la littérature pour désigner une série d'histoires concernant un personnage: par exemple les Voltairiana, Osirisiana, Isisiana etc.

(21) Cette terminologie est celle de C. de Wit qui reprend celle de Rochemonteix. Ce décor, incomplet montre, certes, des offrandes de tissus et de bijoux prophylactiques mais dans un contexte de justification royale. Ils sont un moment des rites funéraires pour Osiris dans sa lecture à l'échelle des Amoniana. A ce titre, ce décor présente des points de comparaison avec celui du temple de Qasr el-Agouz. 
Planche I

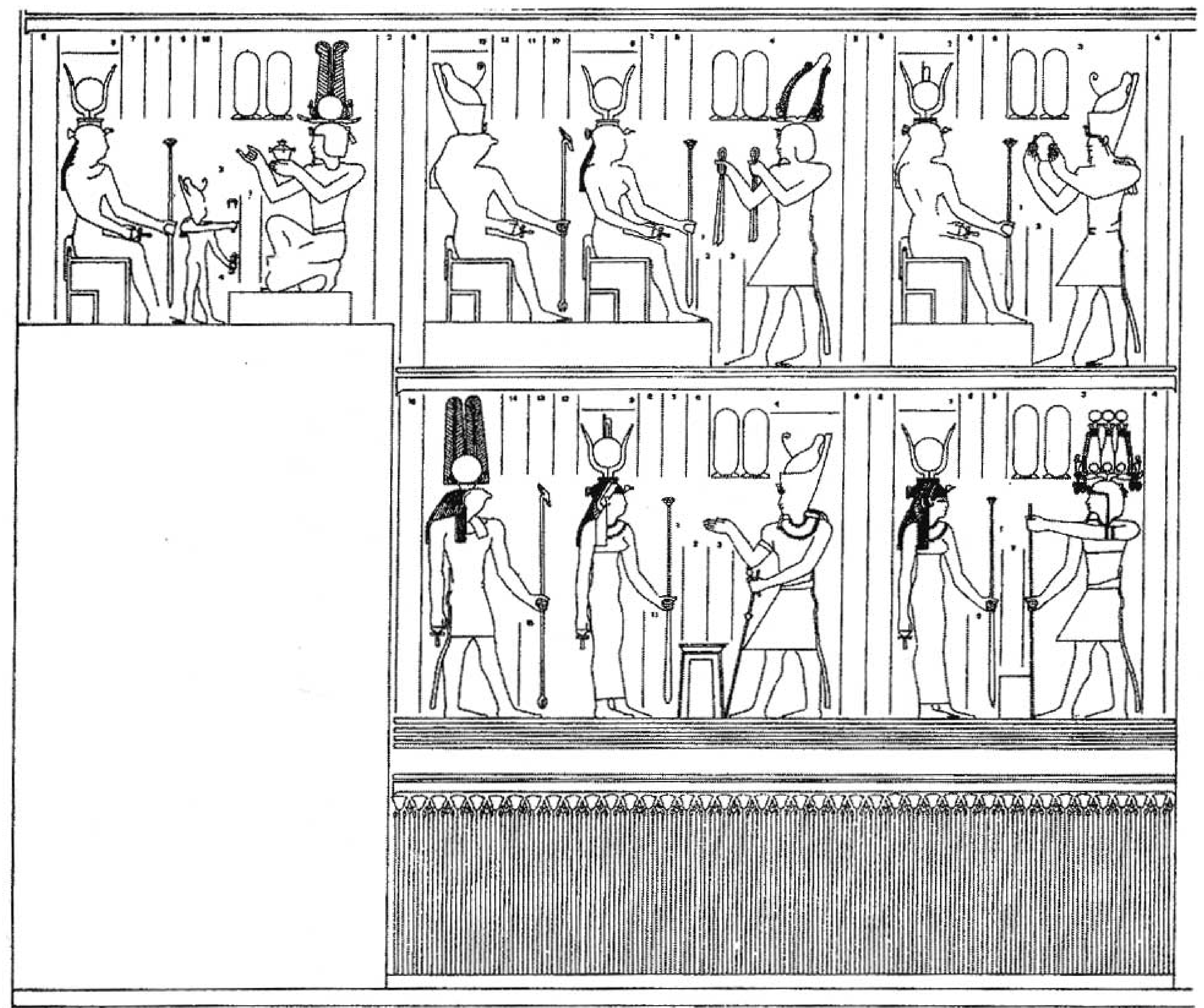

a. Dendara, Salle de l'apparition (dessin B. Lenthérie)

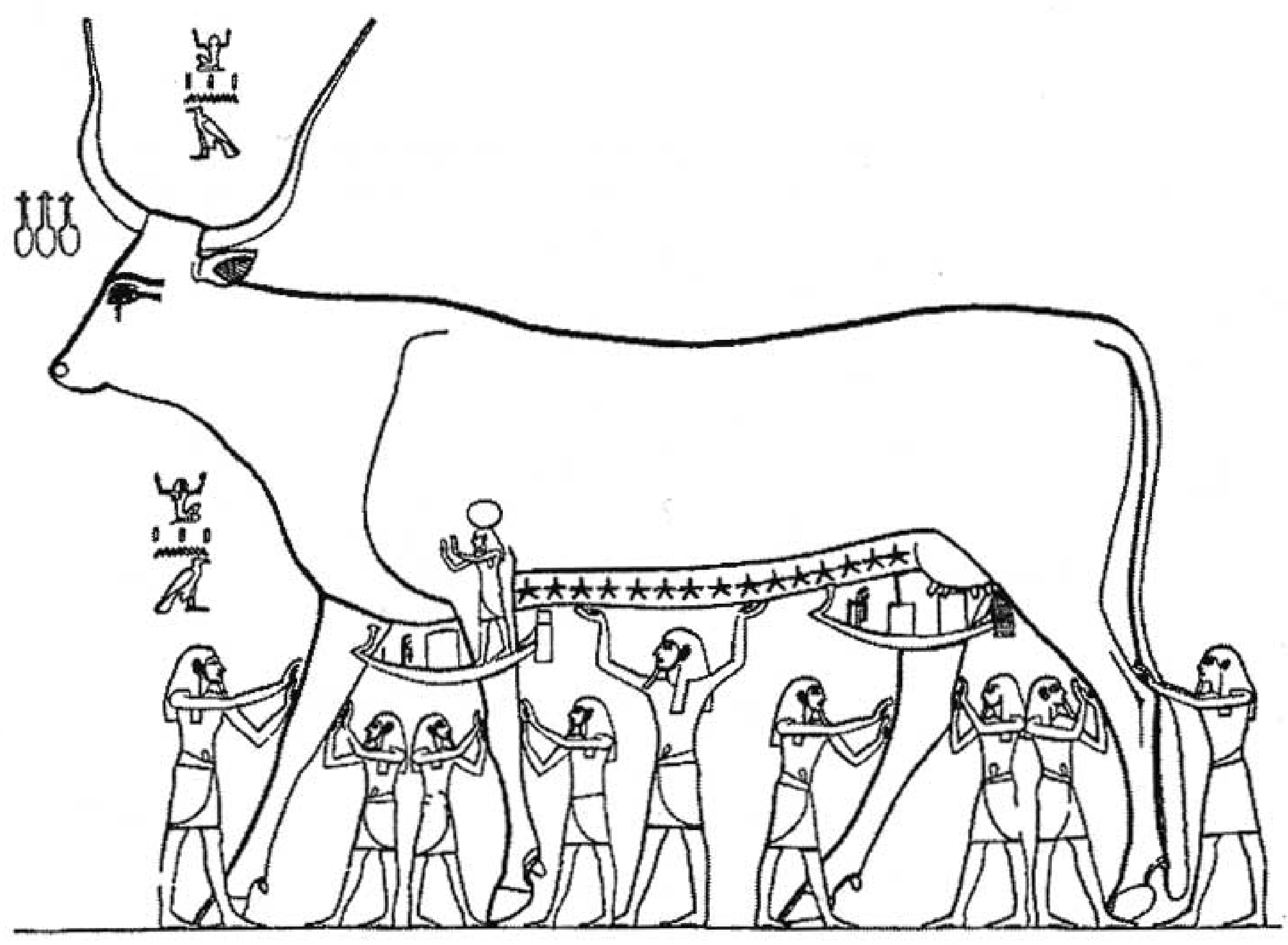

b. La Vache Céleste (chapelle de Toutankhamon) 
Planche II

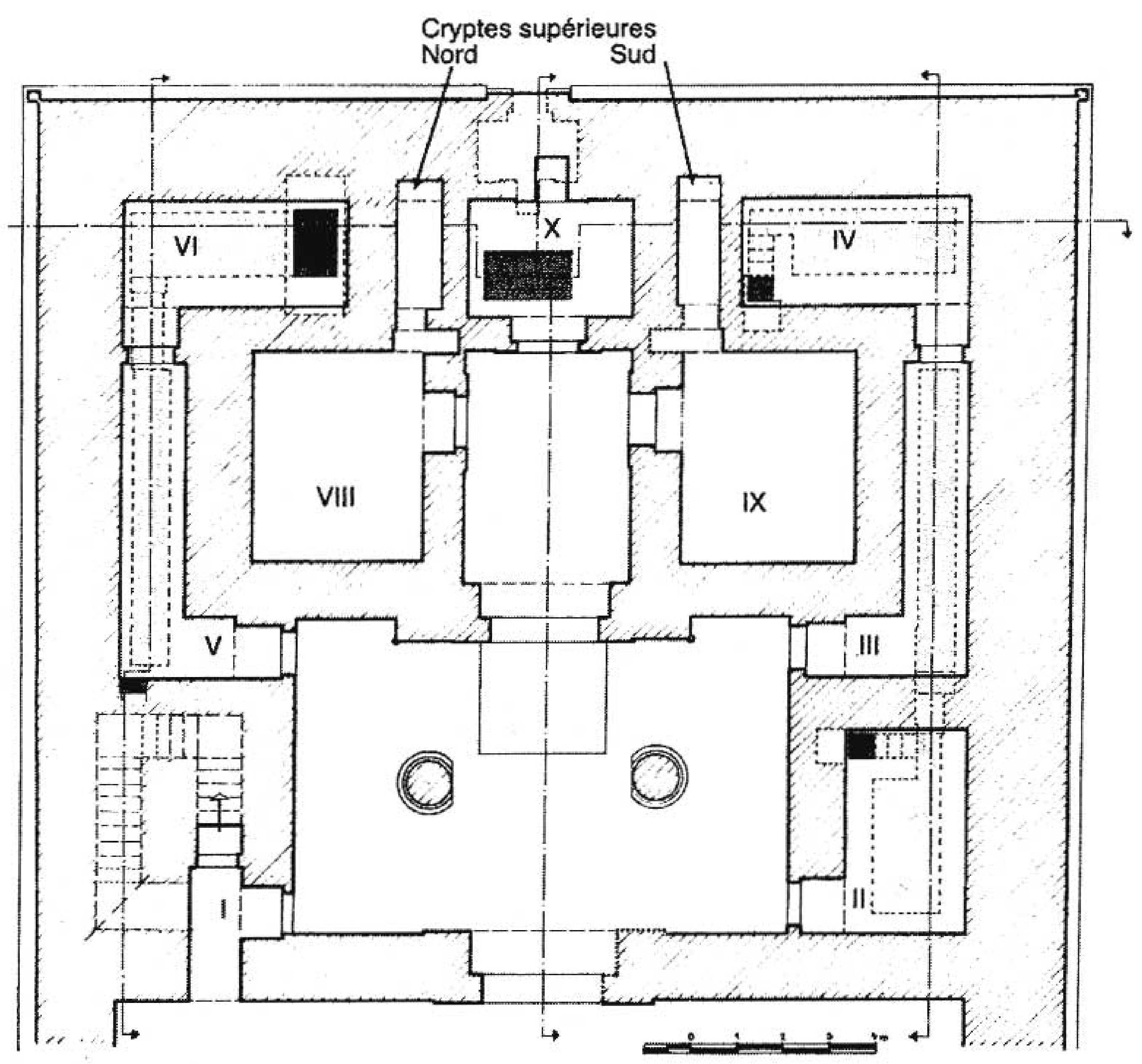

a. Plan du temple d'Opet à Karnak (Relevé C. Traunecker et P. Anus)

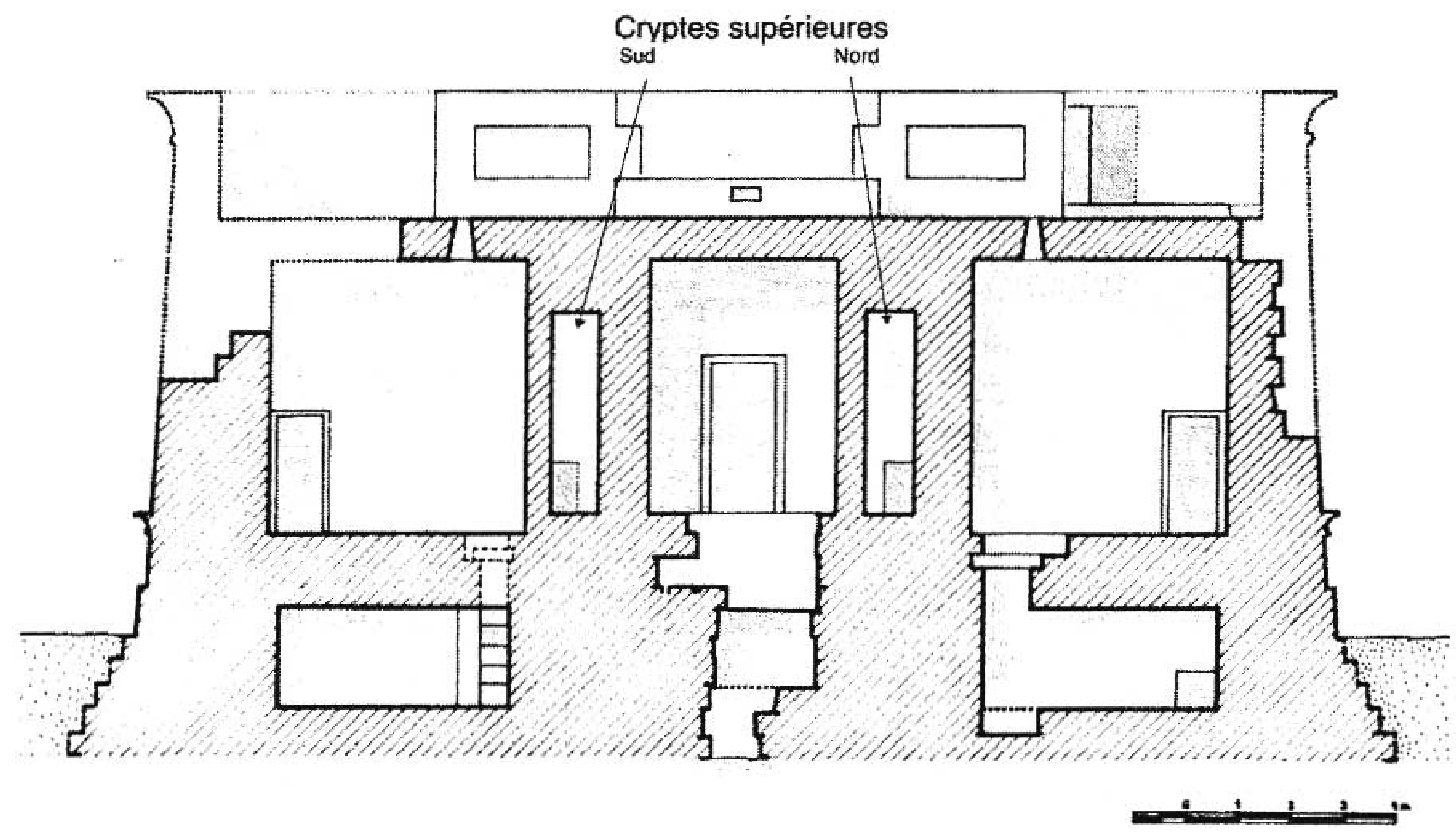

b. Coupe du temple d'Opet sud-nord (Relevé C. Traunecker) 


\section{LES CRYPTES DU TEMPLE D'OPET}

Le temple d'Opet est bâti sur un podium très haut $(2,75 \mathrm{~m})$. Ce dispositif est inhabituel dans l'architecture égyptienne. Dans ce soubassement se cachent quatre appartements secrets: deux au nord (sous la cage d'escalier et sous les salles V et VI), deux au sud (sous la salle II et sous les salles III et IV). Sous le sanctuaire central, un puits contenait probablement un simulacre du tombeau d'Osiris. Ce puits funéraire est en relation liturgique avec une chapelle ménagée dans le soubassement et accessible par l'extérieur du temple (face est). En plus des quatre ensembles de crypte du soubassement, deux cryptes ont été réservées dans l'épaisseur des murs latéraux du sanctuaire central (pl. IIb). On accède à ces deux cryptes supérieures par les salles de culte nord et sud. Leurs entrées sont défendues par un système complexe de blocs coulissants.

La fonction des cryptes de soubassement, malheureusement anépigraphes, est en rapport avec le stockage du matériel de culte spécifique aux deux sanctuaires latéraux: les Osiriana pour la crypte nord-est (le lit de résurrection, soins funéraires à Osiris), les Isiana et Horusiana au sud (langes pour l'enfant divin) au sud.

En novembre 1969, j’ai eu la chance de découvrir des décors peints dans les deux cryptes supérieures $^{22}$. Grâce à une particularité du protocole royal des bandeaux, ces décors ont pu être datés de la dernière partie du règne de Ptolémée VIII Evergète II $^{23}$.

L'intérêt de cette découverte réside dans la rareté des décors de cryptes. Sur la quarantaine de temples à cryptes connus, je n'avais relevé que quatre exemples de cryptes décorées ${ }^{24}$. Depuis, en 1985 , j'ai découvert une nouvelle série de cryptes décorées dans le temple d'el-Qal'a, à Coptos ${ }^{25}$. Les fouilles égyptiennes du temple de Montou à Erment ont mis au jour des pièces souterraines décorées ${ }^{26}$.

On savait déjà par le riche exemple de Dendara que les cryptes pouvaient contenir des statues de culte, soit anciennes, soit encore en usage dans des cérémonies exceptionnelles. L'exemple d'Opet permettait de préciser les fonctions des cryptes dans les temples égyptiens. Dans les cryptes du temple d'Opet, comme à Dendara, certaines des représentations de dieux sont accompagnées de précisions quant à la matière et aux dimensions de l'image de culte. Mais, exceptionnellement, à Opet, certaines des images évoquées sur les parois des cryptes sont représentées à l'échelle $1 / 1$. Ce détail permet d'appréhender la manière dont les Égyptiens mesuraient le corps de leurs dieux.

\section{LES IMAGES DES CRYPTES SUPÉRIEURES DU TEMPLE D'OPET}

Ces cryptes sont d'étroits réduits de $90 \mathrm{~cm}$ de large, profonds de $2,73 \mathrm{~m}$ et hauts de $3,85 \mathrm{~m}$. Les parois des deux cryptes totalisent 16 images divines: 10 au Nord et 6 au Sud. (voir le tableau et la planche III).

(22) C. Traunecker et A. Bellod, "Wandmalereien aus den Krypten der Tempel von Karnak. Erforschung und Fotografie im ultravioletten Licht " Arbeitsblätter für Restauratoren, Trier 7, Heft 1, Gruppe 19, Naturwissenschaftliche Untersuchungen, 1974, 23-2; J. LaUfFray et alii, Kêmi 21, 1971, p. 53-7; idem, Karnak VI, p. 1-65.

(23) C. TrauneckeR, "Cryptes décorées, cryptes anépigraphes» dans Hommages à François Daumas, Montpellier 1986, p. $571-577$

(24) Temples de Sesebi en Nubie, de Mout à Karnak, de Tôd, de Dendara.

(25) L. Pantalacci et C. Traunecker, Le temple d'el-Qala, I et II Le Caire, 1992 et 1998; voir aussi les mêmes auteurs dans BIFAO 93, 1993, p. 379-390.

(26) Ces cryptes sont en cours de publication à l'IFAO par Christophe Thiers et Youri Volokhine: voir BIFAO 103, 2003, p. 562. 
Les deux parois principales de la crypte nord montrent au nord un Osiris ithyphallique qui s'anime sur son lit de résurrection (1). Il est entouré des déesses Isis (2) et Nephthys (3), toutes deux debout, la première à la tête du lit, la seconde à son pied. Sur la paroi sud se dresse un Osiris-Onnophris (4) debout sur un socle élevé, orné d'une figurine de Maât et du roi tenant un grand lotus. En face de lui se déploie la procession des 10 Baou d'Amon (5). Derrière Osiris sont représentées deux enseignes divines: l'une à face hathorique (6), l'autre à face d'hippopotame (7). Sur les petites parois figurent à l'ouest (entrée) les huit dieux primordiaux (8) et sur la paroi du fond une image d'Opet (9) surmonté du Ba d'Amon (10).

Les parois de la crypte sud sont ornées au nord (à gauche) par deux grandes images d'Osiris debout, l'un est Osiris-Onnophris (11), l'autre, Osiris «maître des nourritures» (12). Sur la paroi sud (à droite) Isis assise allaite l'enfant Horus (13). Derrière elle se tient Nephthys (14). Devant Isis, un grand hymne en cinq colonnes exalte le rôle régalien de la déesse ${ }^{27}$. La petite paroi d'entrée, occupée par un grand hymne, n'a pas d'images divines. Sur la paroi du fond figure Opet (15) et, au-dessus d'elle, une image de Ptah (16).

Le statut de ces seize images n'est pas égal. Trois critères permettent de les différencier:

1. Présence d'une indication de matière et de dimensions.

2. Présence d'une légende écrite précisant que la statue est réellement présente dans le temple (texte).

3. Présence d'un doublet de l'image dans une des trois salles de culte.

Le tableau suivant donne l'état, image par image:

\begin{tabular}{|c|l|c|c|c|c|}
\hline \multirow{2}{*}{\multicolumn{2}{|c|}{ IMAGES }} & \multicolumn{2}{c|}{ Cryptes } & \multicolumn{2}{c|}{ Salles de culte } \\
\cline { 3 - 6 } & $\begin{array}{c}\text { Matière et } \\
\text { dimensions }\end{array}$ & Texte & $\begin{array}{c}\text { Présence } \\
\text { doublet }\end{array}$ & $\begin{array}{c}\text { Matière et } \\
\text { dimensions }\end{array}$ \\
\hline Crypte Nord & & & & \\
\hline 1 & Osiris sur son lit & oui & & Opet 120 & oui \\
\hline 2 & Isis & oui & & Opet 120 & oui \\
\hline 3 & Nephthys & oui & & Opet 120 & oui \\
\hline 4 & Osiris-Onnophris & oui & Opet 110 & \\
\hline 5 & Les dix Baou & & & & \\
\hline 6 & Enseigne Hathorique & & & Opet 168 & \\
\hline 7 & Enseigne d'Opet & & & Opet 168 & \\
\hline 8 & Les huit dieux primordiaux & & & Opet 118 & \\
\hline 9 & Opet & oui & & Opet 168 & oui \\
\hline 10 & Le Ba d'Amon & oui & & Opet 120 & \\
\hline & Crypte Sud & & & & \\
\hline 11 & Osiris-Onnophris & & oui & Opet 110 & \\
\hline 12 & Osiris maitre des nourritures & oui & oui & & \\
\hline 13 & Isis allaitant Horus & oui & oui & Opet 110 & \\
\hline 14 & Nephthys & oui & & & \\
\hline 15 & Opet & oui & & Opet 168 & oui \\
\hline 16 & Ptah & oui & & & \\
\hline
\end{tabular}

Onze statues sont représentées avec des indications de matière et de dimensions: dans la crypte nord: l'ensemble d'Osiris sur son lit et des deux déesses qui l'encadrent, la grande statue de bronze noir d'Osiris-Onnophris et, sur la paroi du fond, les images d'Opet et du Ba d'Amon. Dans la crypte sud, toutes les statues représentées sont décrites par la matière et les dimensions sauf l'OsirisOnnophris.

(27) Des passages de cet hymne sont connus par des parallèles dans le temple de Dendara: Dendara II, 200 (1-8) et IV 45 (11-15), 46 (1-4). Voir aussi R. PREYs, Les complexes de la Demeure du Sistre et du Trône de Rê, OLP 106, Leuven 2002, p. 28 et sq. 
On pourrait imaginer que toutes les onze images techniquement décrites étaient réellement présentes dans les deux cryptes. Sur une maquette, nous avons étudié le rangement de ces statues dans ces espaces, mais force fut de constater qu'il est impossible de les placer toutes, en particulier le lit de renaissance d'Osiris, bien trop grand. L'existence de doublets sur les parois des salles de culte, parfois avec des légendes de dimensions, implique la possibilité d'une autre localisation de l'objet dans le temple en particulier dans les cryptes de soubassement.

\section{Les statues réellement présentes dans les cryptes}

Comment identifier parmi les onze statues celles qui étaient réellement présentes dans les cryptes? Tournons-nous vers les textes qui les accompagnent. Normalement ceux-ci soit commencent par la formule traditionnelle "Paroles dites par X», soit énoncent directement le nom de la divinité représentée sans aucune formule d'introduction. Il en est ainsi pour douze des seize images représentées dans les cryptes de culte du temple d'Opet.

Mais, pour quatre d'entre elles (une dans la crypte nord, trois dans la crypte sud), les légendes hiéroglyphiques utilisent un formulaire exceptionnel commençant par un pronom relatif, usage qui n'est pas autorisé par la syntaxe égyptienne. Pour tourner cette difficulté grammaticale, il faut considérer que l'image commentée constitue en elle-même le premier hiéroglyphe de la phrase. De fait, l'image divine à l'échelle $1 / 1$ est un physiogramme ayant la fonction d'un substantif ${ }^{28}$ :

(4) Crypte Nord: Osiris-Onnophris devant les dix Baou d'Amon

\{Cette image\} qui est dressée dans ce temple, (c'est) Osiris-Onnophris, juste de voix [roi des dieux]. Bronze noir, hauteur 3 coudées, 2 palmes, 2 doigts.

(13) Crypte Sud: Isis allaitant Horus

\{Cette image\} qui est dressée dans ce temple, (c'est) Isis la grande, mère divine, œil de Rê, maîtresse du ciel, dame de tous les dieux, vénérable grande dans Thèbes. Bois plaqué d'or hauteur 2 coudés et 2 doigts.

(11) Crypte Sud: Osiris gainé debout \{Cette image\} qui est dressée dans ce temple, (c'est) Osiris, maître des nourritures (kaou), abondant de subsistances (djefaou), ... couronne en or, hauteur totale ( $r$ tep.f) 3 coudées et demie.

(12) Crypte Sud: Osiris gainé debout

\{Cette image\} qui est dressée dans (le temple) d'Opet-la-grande ... au nord, (c'est) Osiris-Onnophris, roi des dieux.

La mention "dans ce temple» implique, à mon avis, que la statue décrite était physiquement présente dans la crypte. Cette interprétation s'appuie, entre autres, sur l'exemple des descriptions des objets des cryptes du temple de Saft el-Henneh. Dans cette perspective, les cryptes supérieures ne contiendraient que trois statues de culte: au nord, un Osiris-Onnophris, au sud, une Isis allaitante et un Osiris «maittre des nourritures».

Reste le cas de la quatrième légende de la crypte sud(12). Cette légende présente trois particularités: d'une part, le lieu cité est le temple d'Opet sans pronom démonstratif, suivi d'un texte mutilé mais donnant probablement des précisions quant à sa localisation dans le temple: [salle] ou [crypte] nord; d'autre part l'épithète et l'iconographie divines reproduisent celles de la statue de la crypte nord (4); enfin il n'est pas fait mention de la matière ni des dimensions de la statue.

(28) Pour des constructions analogues voir: le naos de Saft el-Henneh (Naville, The shrine of Saft el Henneh, pl. 3, lg. 3; pl. 6, lg. 1 et 2); la tablette d'inventaire d'Héliopolis (H. RIскE ZÄS 71, 1935, p. 112) la stèle dite de la fille de Chéops (DARESSY, RT 30, 1908, p. 1.) 
Je propose de voir dans cette image (12) de la crypte sud un simple rappel, un lien iconographique avec la statue (4) présente dans la crypte nord. Concluons:

1. La crypte nord ne contenait qu'une seule statue: le grand Osiris (4) en bronze noir de $1,75 \mathrm{~m}$ de haut, image maitresse du temple d'Opet dans sa moitié nord. C'est en elle que se concentraient les forces universelles des dix Baou d'Amon, expression des puissances qui animent l'univers ${ }^{29}$. La dimension de cette statue et sa matière en font un objet exceptionnel. Rares sont les bronzes égyptiens de cette taille ${ }^{30}$. Tout porte à croire que le bronze était appliqué sur une âme en bois, comme pour les célèbres statues de la VI ${ }^{\mathrm{e}}$ dynastie trouvées à Hiéraconpolis ${ }^{31}$.

2. La crypte sud contenait deux statues: L'image d'Isis allaitant Horus (11) en bois doré de $1,08 \mathrm{~m}$ de haut est l'image de culte maîtresse de la moitié sud du temple consacrée aux Isisana. Elle côtoyait une seconde image d'Osiris «maître des nourritures», elle aussi de grande taille $(1,83 \mathrm{~m})$. La description de sa matière est malheureusement perdue.

\section{LES DIMENSIONS DES STATUES DES CRYPTES DU TEMPLE D'OPET : IMAGE ET RÉALITÉ}

Quel est le rapport entre les dimensions de l'objet et celles de sa représentation? Sur le naos de Saft el-Henneh, les nombreuses images de divinités, qui, d'après les textes, mesuraient une coudée $(52,3 \mathrm{~cm})$, sont nettement plus petites. Dans la crypte de terrasse de Tôd ${ }^{32}$ sont figurées 116 images de divinités sur les deux parois conservées. Leurs dimensions indiquées sont réduites, elles varient de deux palmes $(15 \mathrm{~cm})$ à 4 coudés et 4 palmes, soit $2,40 \mathrm{~m}$, mais, dans leur grande majorité, elles annoncent une taille d'une coudée. Les quatre cryptes à statues, de Dendara ${ }^{33}$ totalisent 284 images divines ${ }^{34} .47$ d'entre elles n'ont pas d'indication descriptive (17\%), 9 ont leur description détruite (3\%), $68(24 \%)$ présentent des dimensions particulières et $160(56 \%)$ mesurent une coudée et sont en or ou en bois plaqué d'or ${ }^{35}$.

Dans une étude récente, F. Hoffmann, reprend la question des dimensions des statues. Pour lui les dimensions des statues relèveraient d'un système de progression mathématique ${ }^{36}$. Il réduit les dimensions en «doigts » et constate dans les chiffres obtenus la présence de série de multiples de 11, $10,15,12,18,16$ etc. Cependant force est de constater l'absence de fréquences significatives pour chacun de ces multiples. Pour les images décrites des cryptes de Dendara, cet auteur pense qu'il

(29) Voir infra, p. 64 et note 37.

(30) La statue de bronze de Pépi I ${ }^{\mathrm{er}}$ mesure $1,77 \mathrm{~m}$ de haut (âme en bois et plaques de bronze). Elle a été restaurée récemment. Le plus grand Osiris de bronze que je connaisse est au Musée de Leyde et mesure 1,06 m (deux coudées). Les "grands » bronzes mesurent $92-96 \mathrm{~cm}$. (ROEDER, JEOL 6, 1939, p. 266.). Sur le bronze noir voir CoONEY, dans ZÄS 93, 1966, p. 43.

(31) Quibell et Green, Hieraconpolis II, p. 46-47, pl. 50-52. Épaisseur du cuivre de 2 à $5 \mathrm{~mm}$. Elles ont été restaurées récemment.

(32) C. THIERs, Tôd. Les inscriptions du temple ptolémaïque et romain, II. Textes et scènes $\mathrm{n}^{\circ} 173-329$, III. Relevé photographique (J.-Fr. Gout), Le Caire 2003.

(33) Est $n^{\circ} 1$, Sud $n^{\circ} 1$, Ouest $n^{\circ} 1$, Est $n^{\circ} 4$.

(34) Dans cette analyse, nous nous sommes borné aux images des cryptes et nous n'avons pas tenu compte des images avec indications de matière et dimension sur les parois des salles de culte du temple.

(35) Sur les statues de culte de Dendara, voir S. Cauville, "Les statues cultuelles de Dendera d'après les inscriptions pariétales», BIFAO 87, 1987, p. 73-117. Pour les statues de culte en général en Égypte ancienne, voir D. LoRTON, "The Theology of Cult Statues in Ancient Egypt" dans Born in Heaven, Made on Earth. The Making of the Cult image in the Ancient Near East édité par M. B. Dick, Winona Lake, Indiana 1999, p. 123-210.

(36) Friedhelm HofmmanN, «Measuring Egyptian Statues», dans Alt Orient und Ancien Testament $\mathrm{n}^{\circ} 297$ «Under our Sky» ed. Steele et Imhausen, Sur ce sujet du même auteur voir son travail inédit d'habilitation: Wort und Bild. Texte und Untersuchungen zur ägyptischen Statuenbeschreibung.Würzburg University, 2001. 
Le décor des crypte supérieures d'Opet

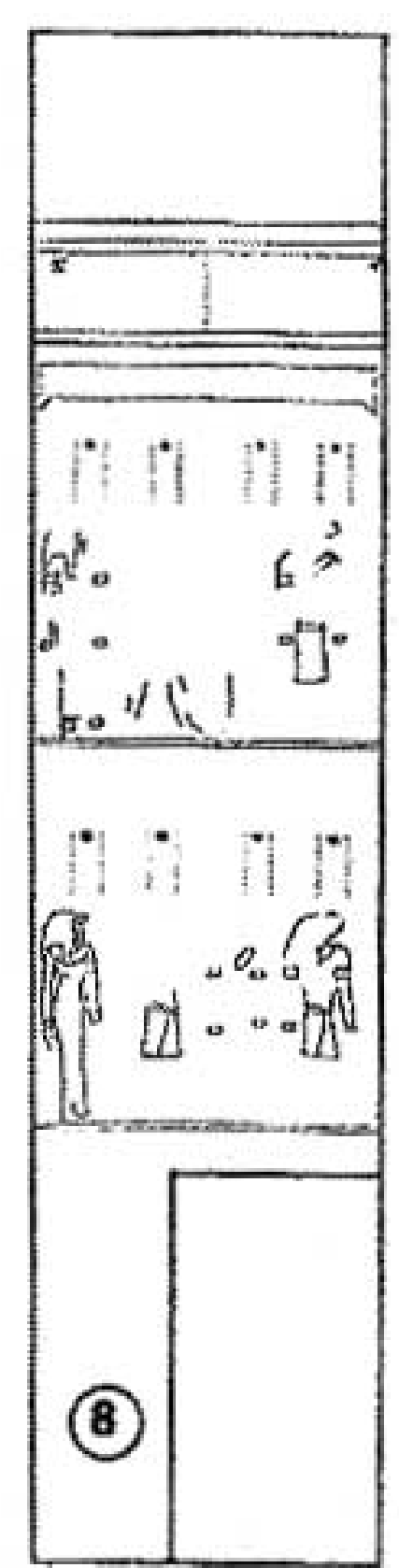

0

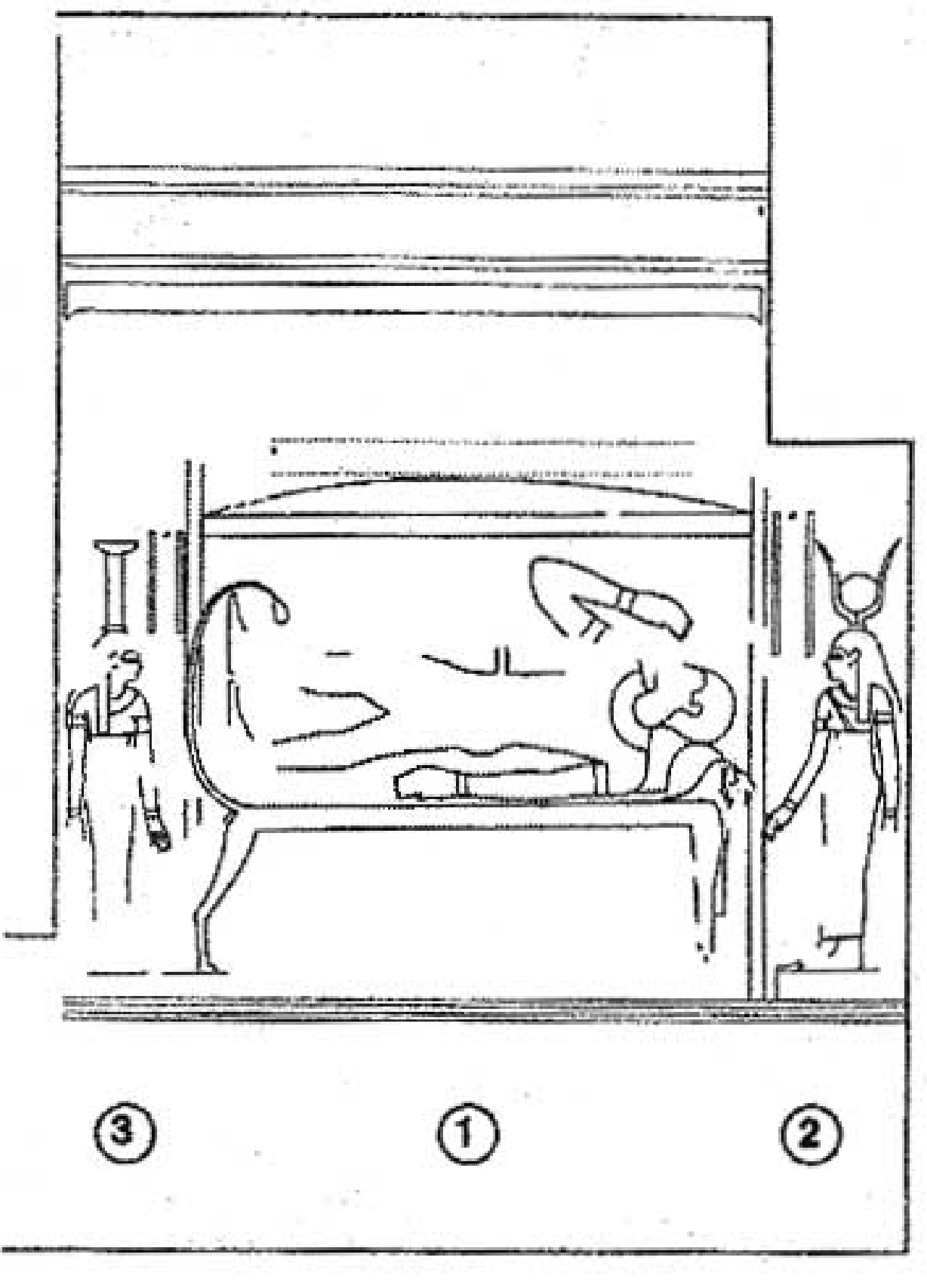

N

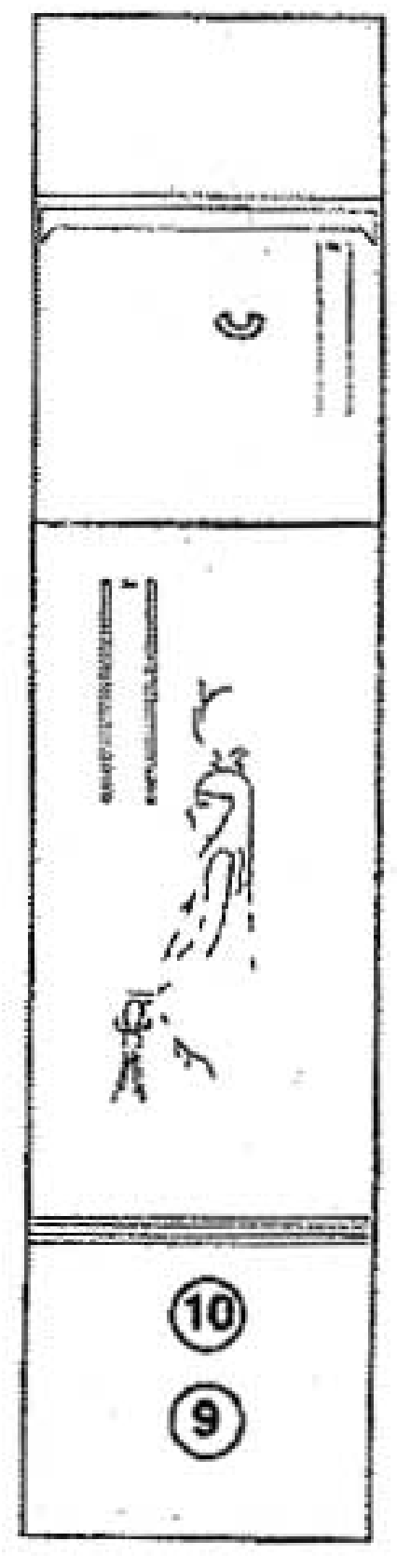

E

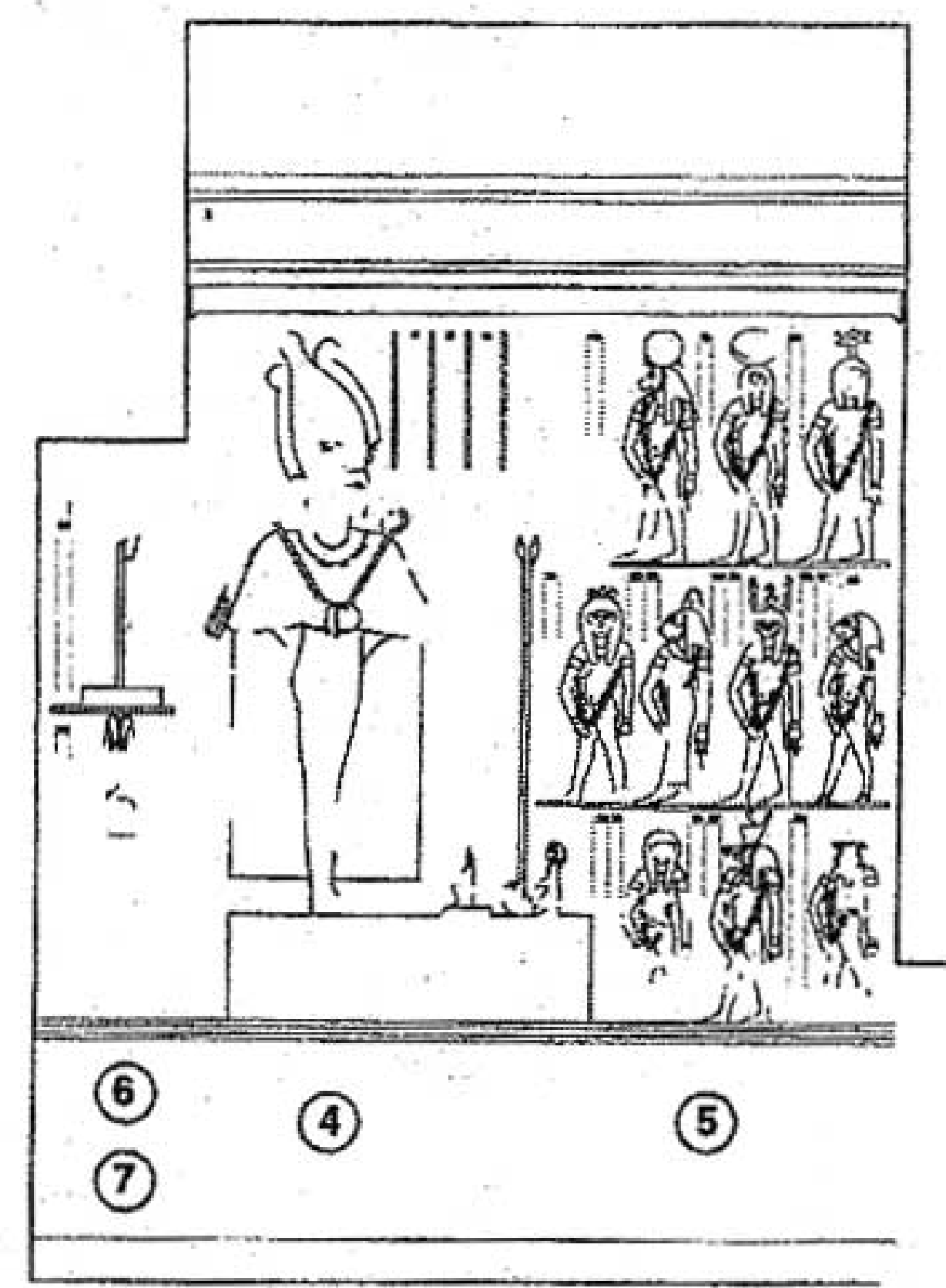

S

a. Crypte nord
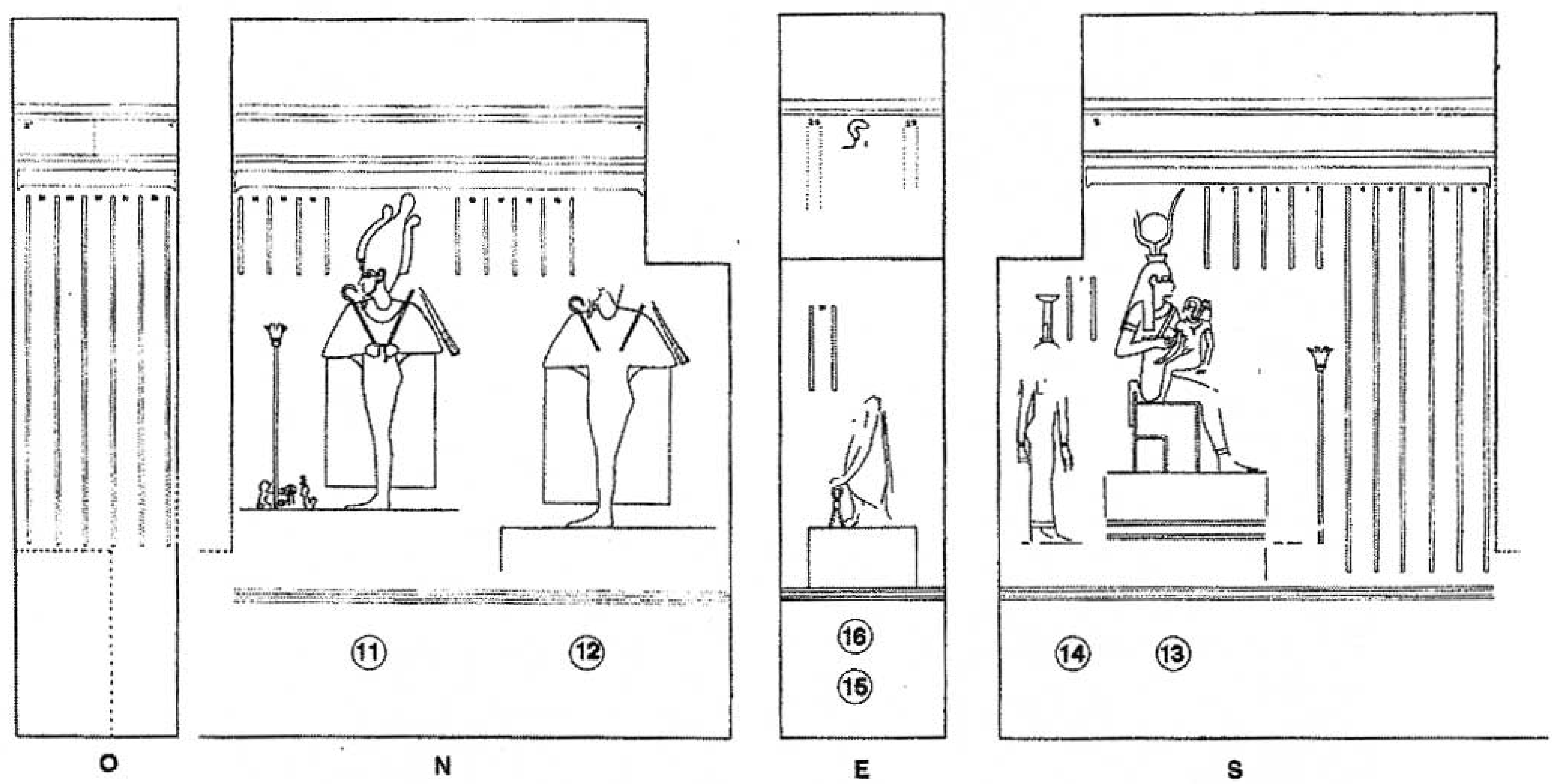

b. Crypte sud 
Planche IV

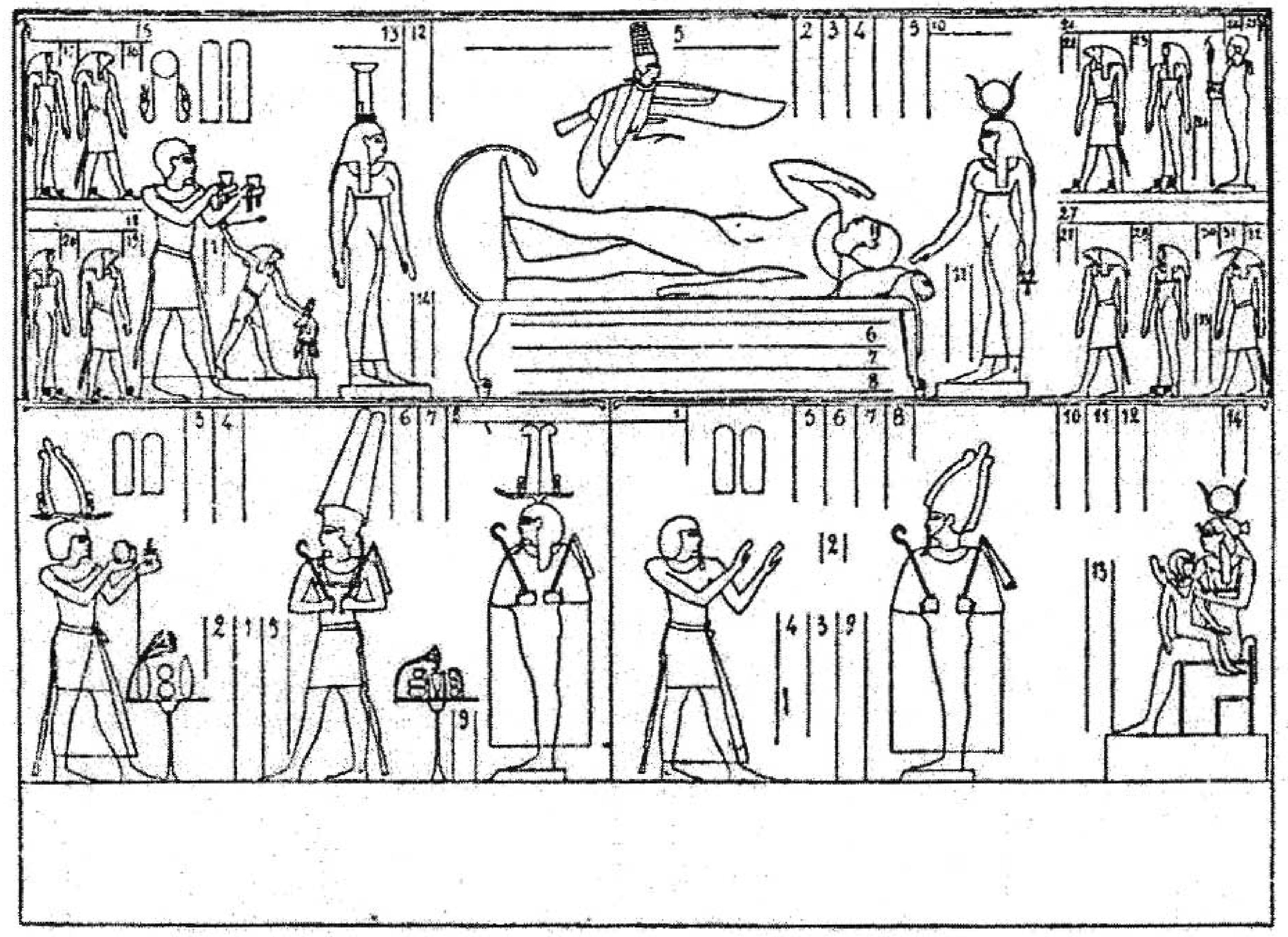

a. Décor de la salle nord, selon Rochemonteix I

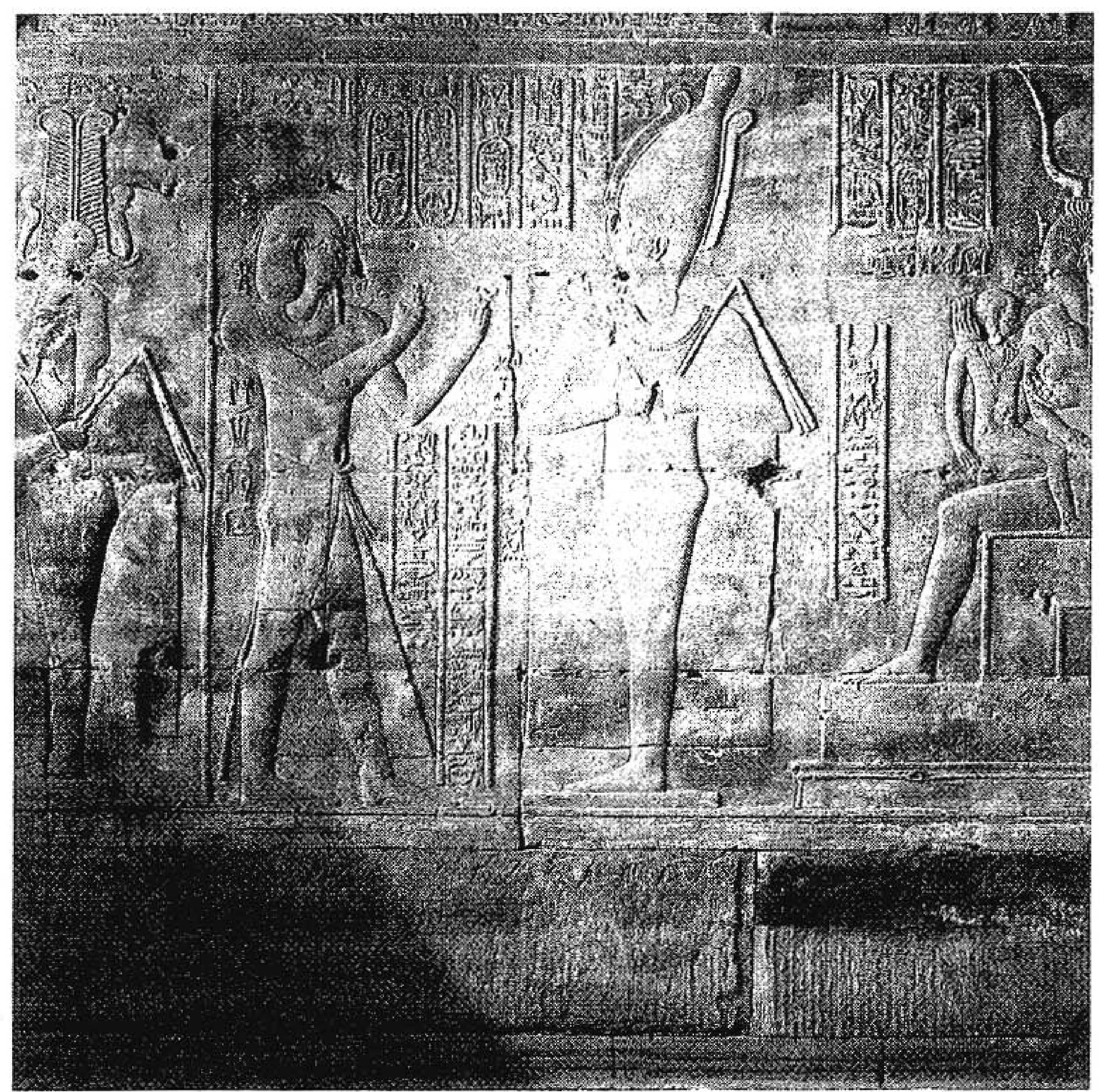

b. Le grand Osiris-Omnophris. A gauche, Osiris-Merty plus petit 
s'agissait d'une sorte d'archivage des caractéristiques des statues de Dendara, servant de modèle aux fabricants de statues du temple et non pas d'images réelles déposées dans ces espaces secrets. L'hypothèse est intéressante mais demanderait à être nuancée. F. Hoffmann suggère également que le terme "qa», "hauteur» désignerait de fait la hauteur de la ligne du regard. Il observe justement que sur le naos de Saft el-Henneh, par exemple, les accessoires (hautes plumes, couronnes et coiffures, oreilles des dieux chiens, armes tenues) ne sont pas pris en compte dans la taille de la statue, comme si seul le corps divin était considéré.

Le dossier d'Opet, avec ses images à l'échelle $1 / 1$ permet d'identifier les "points de mesure» et apporte donc de précieuses indications nouvelles. Que constatons nous?

1. Les dimensions des images d'Osiris couché, d'Isis, de Nephthys, et d'Opet sont prises de la plante des pieds au sommet du crâne.

\begin{tabular}{|c|l|l|l|l|}
\hline$(1)$ & Osiris sur son lit & Bois nebes, pierres précieuses & 3 coudées. $(1,569 \mathrm{~m})$ & mesuré $1,60 \mathrm{~m}$ \\
\hline$(2)$ & Isis près du lit & Bois doré & 2 coudées. $(1,046 \mathrm{~m})$ & mesuré $1,06 \mathrm{~m}$ \\
\hline$(3)$ & Nephthys près du lit & Bois doré & 2 coudées. $(1,046 \mathrm{~m})$ & mesuré $1,06 \mathrm{~m}$ \\
\hline$(9)$ & Opet de la crypte nord & Pierre blanche dorée & 1 coudée $2 / 3 .(0,872 \mathrm{~m})$ & mesuré $0,87 \mathrm{~m}$ \\
\hline$(15)$ & Opet de la crypte sud & Pierre blanche dorée & 1 coudée $2 / 3 .(0,872 \mathrm{~m})$ & mesuré $0,87 \mathrm{~m}$ \\
\hline
\end{tabular}

Le socle n'est pas pris en considération. On ne tient compte ni des couronnes des déesses, ni du signe d'identification de Nephthys. La ligne du regard ne semble jouer aucun rôle. Dans quelques cas, on constate un écart de 2 à $3 \mathrm{~cm}$ entre la mesure et son calcul, écart dû à la phase de mise en peinture de l'ébauche (épaisseurs de traits). L'état de conservation des figures 6, 7, 10 et 16 (enseignes et figure du tympan de fond) ne permet pas de conclure quant à leur échelle, mais la place disponible et les quelques éléments lisibles sont compatibles avec l'échelle 1/1.

Dans la crypte sud, la statue de Nephthys derrière Isis allaitante mesurait d'après sa légende deux coudées et quelques doigts, mais ce dernier chiffre n'est pas conservé. L'image mesure 108,3 $\mathrm{cm}$ selon les critères décrits plus haut, soit la valeur de deux coudées et deux doigts. Les mesures prises sur le doublet de la scène sur la paroi nord de la salle nord donnent approximativement les mêmes résultats $(1,60 \mathrm{~m} ; 1,07 \mathrm{~m} ; 1,07 \mathrm{~m} ; 0,87 \mathrm{~m})$.

2. Les dimensions de l'image assise d'Isis sont prises du niveau de la plante des pieds au sommet du crâne de la déesse sans tenir compte de la couronne dite hathorique ni du socle du trône.

\begin{tabular}{|l|l|l|l|l|}
\hline (13) & Isis allaitante & Bois doré & 2 coudées 2 doigts. $(1,083 \mathrm{~m})$ & mesuré $1,12 \mathrm{~m}$ \\
\hline
\end{tabular}

Le doublet de la salle sud avec $0,94 \mathrm{~m}$ mesuré ne respecte pas cette échelle.

3. Les dimensions des Osiris gainés et coiffés de la couronne blanche sont prises du bas du socle au sommet de la couronne.

\begin{tabular}{|l|l|l|l|l|}
\hline$(4)$ & Osiris-Onnophris & bronze noir & 3 coudées, 2 palmes, 2 doigts $(1,756 \mathrm{~m})$ & mesuré $1,765 \mathrm{~m}$ \\
\hline$(11)$ & Osiris-Onnophris & crypte sud & pas d'indication & mesuré $1,73 \mathrm{~m}$ \\
\hline$(12)$ & Osiris seigneur des nourritures & $\begin{array}{l}\text { matière perdue } \\
\text { couronne en or }\end{array}$ & 3 coudée $1 / 2(1,83 \mathrm{~m})$ & mesuré $1,80 \mathrm{~m}$. \\
\hline
\end{tabular}

En somme, cette manière de considérer les dimensions des images relève d'un pragmatisme bien égyptien et se résume en deux règles:

1. Pour les représentations de dieux debout assis ou couchés et dont le sommet du crâne est visible la mesure est prise de la ligne de base des pieds jusqu'au sommet du crâne sans tenir compte de la position. 
2. Lorsque la couronne portée cache le haut de la tête du personnage et rend difficile l'estimation du sommet du crâne (couronne blanche, atef etc.), la coiffure est prise en compte mais la ligne de base reste la plante des pieds.

$\mathrm{Au}$ terme de cette analyse, il apparaît donc que les cryptes cultuelles du temple d'Opet contenaient trois images sacro-saintes faites de matériaux rares. Elles présentent des mensurations précises qui ne sont pas conventionnelles et sont reproduites à l'échelle $1 / 1$ sur les parois de cryptes. Il s'agit donc de statues réelles et non pas de modèles théoriques.

Les textes qui les accompagnent livrent le secret du système théologique amonien. La puissance de régénération et de résurrection d'Osiris est alimentée par l'énergie créatrice amonienne déclinée et décrite par les dix Baou d'Amon. Ils sont les forces qui animent d'une part lè soleil, la lune, l'air, l'eau et le feu, et celles qui d'autre part habitent les êtres vivants: l'humanité, les quadrupèdes, les oiseaux, les poissons et ceux qui vivent dans le sol ${ }^{37}$. Ainsi selon l'enseignement secret de la crypte nord, la puissance amonienne transcende Osiris. Dans la crypte sud, Isis est la mère attentive de l’héritier divin de la puissance amonienne.

\section{Statues secrètes et dimensions affichées}

Mais les officiants ordinaires et autres serviteurs du temple ignoraient tout de ces textes et images cachés au sein des murs du temple. Les systèmes de fermeture des cryptes se fondaient dans l'appareil des parois et rien ne laissait deviner la présence des pièces secrètes et encore moins l'existence de statues remarquables par leur taille et leurs matériaux. Pourtant l'auteur du décor de la salle nord a introduit dans la composition du premier registre un indice à l'intention d'un observateur attentif pouvant le conduire à s'interroger sur la présence de statues exceptionnelles cachées

Sur la paroi nord, figure au registre supérieur la célèbre scène de la résurrection d'Osiris (pl. IVa). Cette image, répétition de celle de la crypte $(1,2,3,5)$, est accompagnée de mentions de matière et de taille. Le registre inférieur est occupé par deux grandes scènes (direction liturgique vers la droite). La première, à gauche, montre le roi accomplissant fumigation et libation devant Amon et Osiris-Merty (Opet 109). Dans la seconde, (Opet 110) le roi «adore et contente son maître» devant Osiris-Onnophris et Isis assise allaitant son enfant.

L'ancien dessin de Rochemonteix ${ }^{38}$ de cette paroi affiche une composition classique respectant les lois de l'isocéphalie pour les personnages des deux scènes. Mais, sur place, j'ai constaté qu'en réalité les deux images divines de la scène de droite (Opet 110) sont nettement plus grandes que celles du reste du registre (pl. IVb). Dans la scène de gauche (Opet 109), la taille des dieux (plante des pieds au sommet du crâne) est de $1,18 \mathrm{~m}^{39}$. La scène de droite (Opet 110) détonne par la taille exceptionnelle des images divines: $1,75 \mathrm{~m}$ pour Osiris-Onnophris, couronne blanche comprise, et $1,12 \mathrm{~m}$ pour Isis assise et allaitante (de la plante des pieds au sommet du crâne) ${ }^{40}$. Les épithètes d'Osiris et d'Isis qui accompagnent ces deux images sont identiques à celles des cryptes mais ici le texte garde le silence sur la matière et la taille. Apparemment, ces images se présentent comme de simples évocations d'êtres divins ${ }^{41}$ et non comme des reproductions d'objets de culte. Or les

(37) C. Traunecker, Les dieux de l'Égypte, coll. Que Sais-Je, Paris 1992, p. 96-97.

(38) Ce dessin est actuellement le seul publié: Maxence de RochemonteIX, "Le temple d'Apet où est engendré l'Osiris de Thèbes» dans RT 3, 1882, p. 72-86 et 6, 1885, p. 21-35. Voir aussi A. BAILlET, «Le temple d'Apet à Carnac» dans RT 20,1898, p.100-111. Il est utilisé dans l'édition de C. de WITT 1962 (cf. supra, note 17).

(39) Tout les dieux et officiants du premier registre, hormis la scène 110, sont de ce gabarit. L'Osiris-Merty de la scène 109 est coiffé d'une double plume culminant à $1,59 \mathrm{~m}$.

(40) La même image de culte sur la paroi sud de la salle sud ne mesure que $0,94 \mathrm{~m}$. 
dimensions des images visibles reproduisent exactement celles des statues secrètes. Tout se passe comme si les décorateurs voulaient signaler discrètement aux initiés l'existence de statues sacrosaintes cachées quelque part dans les entrailles du temple.

\section{LE TEMPLE INTERMITTENT}

Ces constatations m'ont conduit à envisager un fonctionnement particulier du temple d'Opet et probablement de plusieurs temples de même époque. Ces édifices hautement spécialisés étaient le cadre de célébrations spectaculaires mais rares. Je pense qu'entre ces célébrations commémoratives et ponctuelles, le matériel de culte (statues et meubles) destiné à reconstituer les phases du mythe dans les salles correspondantes dormait en sécurité dans le secret des cryptes de soubassement. En revanche, dans l'obscurité des deux cryptes supérieures, les images sacro-saintes garantissaient la permanence hiérophanique. Un culte minimum était assuré dans le sanctuaire central au cours de l'année mais le temple était en quelque sorte en sommeil. Lors des grandes fêtes osiriennes, on le réveillait: les blocs masquant l'ouverture des cryptes étaient descellés, les verrous repoussés, les blocs mobiles sur roulettes repoussés dans leur logement, le précieux matériel sorti de sa retraite, statues et meubles remontés dans leurs espaces festifs pour une mise en scène liturgique somptueuse ${ }^{42}$.

Aujourd'hui, le grand Osiris-Onnophris de bronze noir, précieux réceptacle de la puissance universelle d'Amon a été arraché de sa crypte, mais sur une paroi voisine maculée de suie et de déjections de chauve-souris, son image de trois coudées deux palmes et deux doigts domine ses voisines et continue à veiller sur le temple.

Claude TraunEcker, UMR 7044, Université Marc Bloch - Strasbourg II

(41) Pour une classification des images sur les parois des temples voir mes «Observations sur le décor des temples égyptiens" dans L'Image et la production du Sacré, sous la direction de F. DunAND, J.-M. SPIESER, J. WirTh, Colloque de Strasbourg 1988, 1991 p.77-101.

(42) Les cryptes inférieures sont anépigraphes sauf quelques graffitis démotiques. Leurs auteurs sont des prêtres commémorant leur descente dans ces lieux secrets, probablement à l'occasion de la sortie annuelle du matériel festif: C. Traunecker, Les cryptes du temple d'Opet à Karnak (mémoire inédit de l'EPHE) p. 58, (crypte Sud-Est). W. SPIEgelberG, ASAE 3, 1902, p. 89-91 (crypte nord-ouest). 